\title{
The $9^{+}$-Intersection: A Universal Framework for Modeling Topological Relations
}

\author{
Yohei Kurata \\ SFB/TR8 Spatial Cognition, Universität Bremen \\ Postfach 330 440, 28334 Bremen, Germany \\ ykurata@informatik.uni-bremen.de
}

\begin{abstract}
The $9^{+}$-intersection is an extension of the 9-intersection, which distinguishes the topological relations between various spatial objects by the pattern of a nested matrix. This paper develops a small set of constraints on this matrix, which is applicable to arbitrary pairs of spatial objects in various spaces. Based on this set of universal constraints, the sets of matrix patterns, each representing a candidate for topological relations, are derived for every possible pair of basic objects (points, directed/non-directed line segments, regions, and bodies) embedded in $\mathbf{R}^{1}, \mathbf{R}^{2}, \mathbf{R}^{3}, \mathbf{S}^{1}$, and $\mathbf{S}^{2}$. The derived sets of candidates are consistent with the sets of topological relations ever identified, as well as yield the identification of some missing sets of topological relations. Finally, the topological relations between a region and a region with a hole in $\mathbf{R}^{2}$ and $\mathbf{S}^{2}$ are identified to demonstrate the applicability of our approach to deriving topological relations between more complicated objects.
\end{abstract}

\section{Introduction}

Topological relations between two spatial objects, which concern how the objects intersect with each other, have been studied extensively in the geographic database community, motivated by the necessity of a formal and cognitively-adequate basis of spatial query language. Previous studies have identified sets of all possible topological relations between various pairs of objects in $\mathbf{R}^{2}$ [1-7], as well as in $\mathbf{R}^{1}$ [8], $\mathbf{R}^{3}$ [9], $\mathbf{S}^{1}$ [10], $\mathbf{S}^{2}$ [11], and $\mathbf{Z}^{2}$ [12]. $\mathbf{R}^{n}$ is an $n$-dimensional Euclidean space, $\mathbf{S}^{1}$ is a circle (1-sphere), and $\mathbf{S}^{2}$ is an ordinary sphere (2-sphere), and $\mathbf{Z}^{2}$ is a discrete raster space. Each identified set of topological relations has a specific practical value. For instance, topological line-region relations in $\mathbf{R}^{2}$ are useful for modeling spatial predicates related to motions, such as enter and go across [13], and topological lineline relations in $\mathbf{R}^{1}$ or $\mathbf{S}^{1}$ are useful for modeling temporal relations $[8,10]$.

As a formal model of topological relations, many studies have adopted the 4intersection [1], the 9-intersection [2], or their extension [5, 6, 10, 14]. In these models, topological relations between two objects $A$ and $B$ are represented by the patterns of a matrix, whose elements represent the intersections between point sets associated with $A$ and those associated with $B$. Usually, candidates for possible topological relations between $A$ and $B$ are derived computationally as the set of matrix patterns that satisfy certain constraints. The set of candidates, each with at least one 
geometric realization, is approved as the set of all possible topological relations between $A$ and $B$. Previous studies developed such a constraint set on the matrix for each pair of objects in each space [1, 2, 4-7, 9-12]. Although there are certain overlaps between such constraint sets [2,9], it is a hard step to develop a constraint set in order to study a new set of topological relations. As a solution to this problem, this paper develops a set of universal constraints, which is applicable to arbitrary pairs of objects embedded in various spaces.

Our universal constraints are applied to the matrix of the $9^{+}$-intersection [6]. The $9^{+}$-intersection is an extension of the 9-intersection, which supports the subdivision of objects' interior, boundary, and exterior. For instance, the exterior of a region with a hole is subdivided into outer and inner exterior subsets. Thanks to the support of such subdivisions, the $9^{+}$-intersection is able to capture the topological relations between various spatial objects, including complicated ones. Accordingly, making use of the $9^{+}$-intersection together with the set of universal constraints, we can easily derive the candidates for the topological relations between various pair of objects embedded in various spaces. Indeed, this paper derives the sets of such candidates for every possible pair of basic objects (points, directed/non-directed lines, regions, and bodies) embedded in $\mathbf{R}^{1}, \mathbf{R}^{2}, \mathbf{R}^{3}, \mathbf{S}^{1}$, and $\mathbf{S}^{2}$. In this paper, lines and regions normally refer to simple lines and simple regions $[4,15]$, respectively. Simple lines are lines with no self-intersection and no branch, derived by a one-to-one mapping from $[0,1]$ to the space. If their two endpoints are ordered, lines are called DLines (directed lines). Simple regions are two-dimensional point sets with a connected interior, no hole, and no spike, as well as no fin in $\mathbf{R}^{3}$. Finally, bodies refer to simple bodies, which are three-dimensional counterparts of simple regions.

The remainder of this paper is structured as follows: Section 2 introduces the $9^{+}$intersection and its matrix-based representation. Section 3 develops a set of universal constraints on the matrix of the $9^{+}$-intersection. Based on this set of constraints, Section 4 derives the candidates for topological relations between every pair of basic objects and analyzes the candidates in comparison with the topological relations identified in the previous studies. Section 5 derives further candidates of basic topological relations by converting the matrix patterns derived in Section 4 and analyzes these new candidates. Section 6 demonstrates the applicability of our approach to the derivation of topological relations between more complicated objects. Finally, Section 7 concludes with the discussion of a future problem.

\section{The $\mathbf{9}^{+}$-Intersection}

The 9-intersection [2] is a model of topological relations between two spatial objects. Based on point-set topology [16], this model distinguishes the interior, boundary, and exterior of each object, which are also called the object's topological parts. Let $X$ be a spatial object and $\bar{X}$ be $X$ 's closure (the intersection of all closed point sets that contain $X$ ). Uppercase letters are used because spatial objects are considered sets of points. $X^{\prime}$ 's interior $X^{\circ}$ is the union of all open sets contained in $X, X^{\prime}$ 's

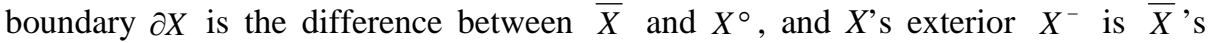
complement. Accordingly, the boundary of a region refers to its looped edge, the 
boundary of a line refers to its two endpoints, and the boundary of a point refers to the point itself.

The 9-intersection captures the topological relation between two spatial objects $A$ and $B$ based on the intersections of $A$ 's three topological parts and B's three topological parts. These $3 \times 3=9$ types of intersections are concisely represented by the 9-intersection matrix in Eqn. 1. Normally, topological relations are distinguished by the presence or absence of these nine types of intersections. The use of some additional properties of the intersections are also proposed for more detailed distinction of topological relations $[14,17]$.

$$
M(A, B)=\left(\begin{array}{lll}
A^{\circ} \cap B^{\circ} & A^{\circ} \cap \partial B & A^{\circ} \cap B^{-} \\
\partial A \cap B^{\circ} & \partial A \cap \partial B & \partial A \cap B^{-} \\
A^{-} \cap B^{\circ} & A^{-} \cap \partial B & A^{-} \cap B^{-}
\end{array}\right)
$$

Each topological part of a spatial object may be subdivided into multiple subparts based on their disconnection or qualitative difference (e.g., dimensions [18]). For instance, the boundary of a DLine is subdivided into two subparts; start-point and end-point [5]. In order to support such subdivision of objects' topological parts, the $9^{+}$-intersection [6] extends the 9-intersection, considering the intersections between the subparts of $A$ 's three topological parts and those of $B$ 's three topological parts. In this model, the topological relations between $A$ and $B$ are characterized by the $9^{+}$intersection matrix in Eqn. 2, whose nine bracketed elements are matrices by themselves, each representing the intersections between the subparts of $A$ 's one topological part and those of $B$ 's one topological part. $A^{\circ_{i}}, \partial_{i} A$, and $A^{-i}$ are the $i^{\text {th }}$ subpart of $A^{\text {'s }}$ interior, boundary, and exterior, while $B^{{ }^{\circ} j}, \partial_{j} B$, and $B^{j_{j}}$ are the $j^{\text {th }}$ subpart of $B$ 's interior, boundary, and exterior, respectively. If a topological part is not subdivided, we consider that this topological part consists of a single subpart. As seen from the comparison of the matrices in Eqns. 1-2, the $9^{+}$-intersection matrix keeps the framework of the 9-intersection matrix; that is, the nine inner matrices in the $9^{+}$intersection matrix uniquely correspond to the nine elements in the 9-intersection matrix. Just like the 9-intersection, topological relations between $A$ and $B$ are distinguished by the presence or absence of all intersections listed in the matrix.

$$
M^{+}(A, B)=\left(\begin{array}{lll}
{\left[A^{o_{i}} \cap B^{o_{j}}\right]} & {\left[A^{o_{i}} \cap \partial_{j} B\right]} & {\left[A^{o_{i}} \cap B^{-j}\right]} \\
{\left[\partial_{i} A \cap B^{o_{j}}\right]} & {\left[\partial_{i} A \cap \partial_{j} B\right]} & {\left[\partial_{i} A \cap B^{-j}\right]} \\
{\left[A^{-i} \cap B^{o_{j}}\right]} & {\left[A^{-i} \cap \partial_{j} B\right]} & {\left[A^{-i} \cap B^{-j}\right]}
\end{array}\right)
$$

As an example, Eqn. 3 shows the $9^{+}$-intersection matrix for the topological relation between a DLine $D$ and a region $R$. In this matrix, $\partial_{1} D$ and $\partial_{2} D$ represent $D$ 's start-point and end-point, respectively. For simplification, if a topological part consists of a single subpart, the subscript assigned to this subpart is omitted. In addition, brackets of inner matrices are omitted if they have only one element. Originally, the $9^{+}$-intersection matrix was introduced to capture such topological DLine-region relations in [6], where 26 relations are identified using the specific 
constraints on the patterns of the $9^{+}$-intersection matrix in Eqn. 3 , instead of the universal constraints proposed in this paper.

$$
\left.M^{+}(D, R)=\left(\begin{array}{ccc}
D^{\circ} \cap R^{\circ} & D^{\circ} \cap \partial R & D^{\circ} \cap R^{-} \\
{\left[\begin{array}{c}
\partial_{1} D \cap R^{\circ} \\
\partial_{2} D \cap R^{\circ}
\end{array}\right]} & \left.\begin{array}{c}
\partial_{1} D \cap \partial R \\
\partial_{2} D \cap \partial R
\end{array}\right] \\
D^{-} \cap R^{\circ} & D^{-} \cap \partial R & \partial_{1} D \cap R^{-} \\
\partial_{2} D \cap R^{-}
\end{array}\right]\right)
$$

For visualization, the patterns of the $9-9^{+}$-intersection matrix are represented by bitmap-like icons [6, 19]. Each icon is partitioned into nine blocks, which correspond to the nine elements of the 9-intersection matrix or the nine element sets of the $9^{+}$intersection matrix (Figs. 1a-b). In the icon of the $9^{+}$-intersection matrix, each block of the icon is further partitioned if the corresponding element set has multiple elements (Fig. 1b). Each block or sub-block is marked out if the corresponding intersection is non-empty. Accordingly, topological relations are distinguished by the icons' marking patterns.

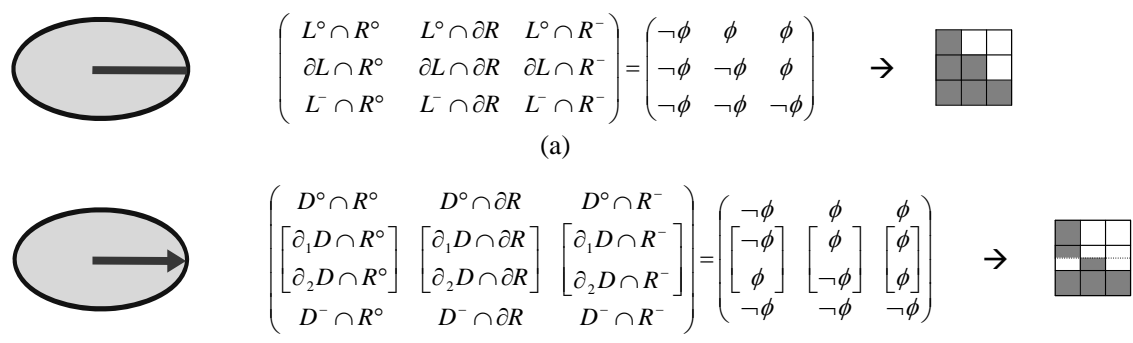

(b)

Fig. 1. Iconic representations of (a) the pattern of the 9-intersection matrix and (b) that of the $9^{+}$-intersection matrix.

\section{Universal Constraints on the $\mathbf{9}^{+}$-Intersection Matrix}

The primitives of a spatial object $X$ are defined as the subparts of $X$ 's interior, boundary, or exterior that are each self-connected and mutually disjoint. For instance, the interior, boundary, and exterior of a DLine in $\mathbf{R}^{1}$ consist of one, two, and two primitives, respectively, as its boundary consists of two distinctive points (i.e., startpoint and end-point) and its exterior consists of two distinctive half-lines (i.e., front and back exterior subparts). Primitives of spatial objects are classified by their dimension and spatial extent (Table 1). For instance, the primitives that form the interior, boundary, and exterior of the DLine in $\mathbf{R}^{1}$ are classified into $B-1 D$ (bounded, non-looped one-dimensional primitive), $O D$ (zero-dimensional primitive), and $U$ - $1 D$ (unbounded one-dimensional primitive), respectively.

By illustrating the class of all primitives that form the interior, boundary, and exterior of a spatial object $X$, as well as the adjacency among these primitives, $X$ 's topological structure is represented as a graph. For instance, Fig. 2 illustrates the 
topological structures of points, DLines, and regions embedded in $\mathbf{R}^{1}, \mathbf{R}^{2}, \mathbf{R}^{3}, \mathbf{S}^{1}$, and $\mathbf{S}^{2}$. These topological structures have the following features:

- Every primitive has at least one adjacent primitive; and

- Every pair of adjacent primitives has different dimensions and belongs to different topological parts.

Table 1. Classes of basic primitives.

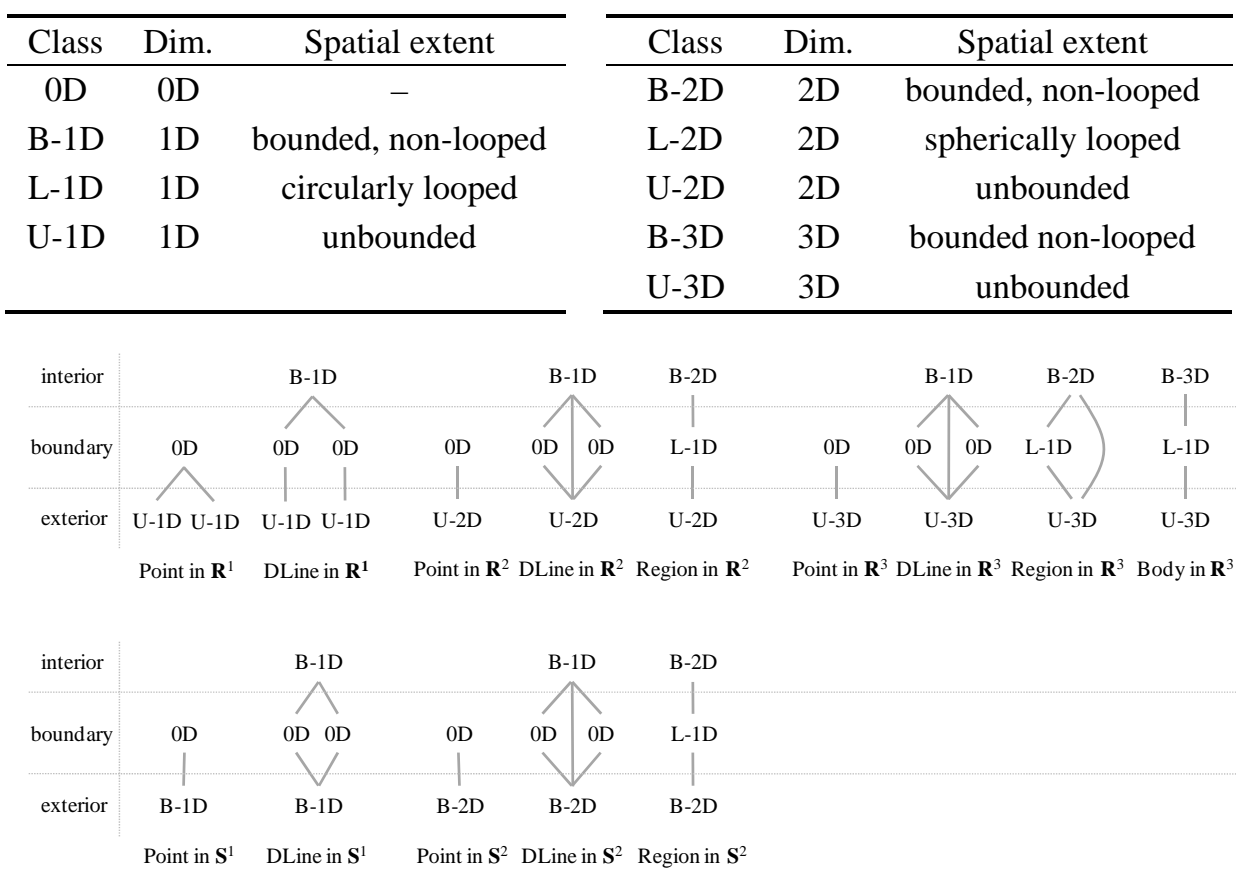

Fig. 2. Topological structures of points, DLines, and regions embedded in $\mathbf{R}^{1}, \mathbf{R}^{2}, \mathbf{R}^{3}, \mathbf{S}^{1}$, and $\mathbf{S}^{2}$, based on the class and adjacency of their primitives.

Assume that two spatial objects $A$ and $B$ are embedded in the space $S$. Then, $A$ 's primitives and $B$ 's primitives must satisfy the following nine conditions (see Appendix for their proofs):

- Condition 1: Each of A's primitives intersects with at least one of B's primitives, and vice versa.

- Condition 2: If $A$ has a zero-dimensional primitive (OD), then it intersects with only one of $B$ 's primitives, and vice versa.

- Condition 3: If $A$ 's primitive $P_{i}$ intersects with more than one of $B$ 's primitives, then these primitives jointly form a connected point set, and vice versa.

- Condition 4: A's bounded primitives, either looped or non-looped, cannot contain a $B$ 's unbounded primitives, and vice versa. 
- Condition 5: A's non-looped primitives cannot contain a B's looped primitive of the same dimension, and vice versa.

- Condition 6: If $A$ 's primitive $P_{i}$ intersects with $B$ 's primitive $Q_{j}$, then all of $P_{i}$ 's adjacent higher-dimensional primitives intersect with $Q_{j}$ or at least one of $Q_{j}$ 's adjacent higher-dimensional primitives, and vice versa (Fig. 3a).

- Condition 7: If $A$ 's primitive $P_{i}$ is contained by $B$ 's primitive $Q_{j}$ and they belong to the same primitive class, then at least one of $P_{i}$ 's adjacent lower-dimensional primitives intersects with $Q_{j}$, and vice versa (Fig. 3b).

- Condition 8: If $A$ 's primitive $P_{i}$ intersects with $B$ 's primitive $Q_{j}$ that is lower dimensional than $P_{i}$, then $P_{i}$ intersects with at least one of $Q_{j}$ 's adjacent higherdimensional primitives (Fig. 3c) or at least two of them including one of $Q_{j}$ 's adjacent primitives that are bounded, non-looped, and one-dimensionally higher than $Q_{j}$ (Fig. 3d), and vice versa.

- Condition 9: If $A$ has only one unbounded primitive whose dimension is the same with $S$, then this primitive intersects with all of $B$ 's unbounded primitives, and vice versa.

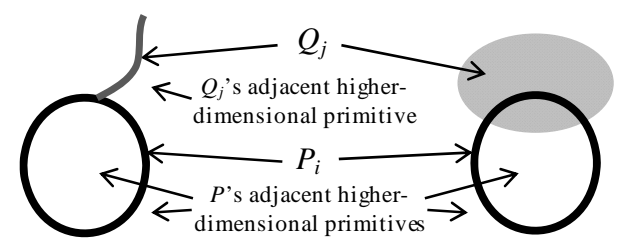

(a)

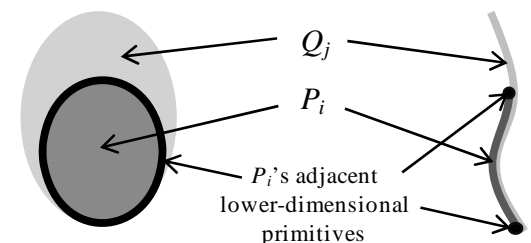

(b)

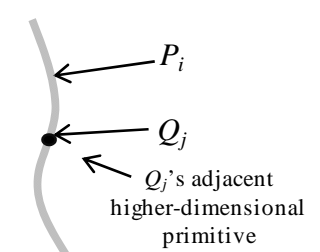

(c)

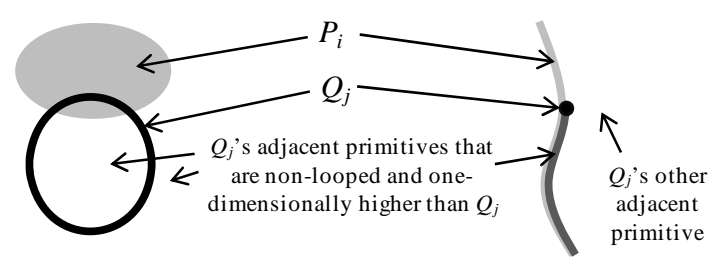

(d)

Fig. 3. Intersection of two primitives $P_{i}$ and $Q_{j}$ determines the presence of intersections of $P_{i}$ 's certain adjacent primitives and $Q_{j}$ or $Q_{j}$ 's adjacent primitives

When the $9^{+}$-intersection illustrates the intersections of $A^{\prime}$ 's primitives and $B$ 's primitives, Conditions 1-9 serve as the constraints on the patterns of the $9^{+}$intersection matrix. For instance, the $9^{+}$-intersection matrix in Eqn. 3 illustrates the intersections of the primitives of a DLine $D\left(D^{\circ}, \partial_{1} D, \partial_{2} D\right.$ and $\left.D^{-}\right)$and the primitives of a region $R\left(R^{\circ}, \partial R\right.$ and $\left.R^{-}\right)$. This matrix may distinguish 4096 patterns, as it has $4 \times 3$ two-valued elements, but only 26 patterns among the 4096 patterns satisfy Conditions 1-9. Consequently, we can conclude that these 26 patterns of the $9^{+}$-intersection matrix represent the candidates for the topological relations between a DLine $D$ and a region $R$. 


\section{Deriving Topological Relations between Basic Objects}

For every possible pair of basic objects (points, DLines, regions, and bodies) embedded in $\mathbf{R}^{1}, \mathbf{R}^{2}, \mathbf{R}^{3}, \mathbf{S}^{1}$, and $\mathbf{S}^{2}$, we derived the patterns of the $9^{+}$-intersection matrix that satisfy Conditions 1-9, making use of their structural information illustrated in Fig. 2. Table 2 shows the number of the derived matrix patterns. Since each matrix pattern represents a candidate for topological relations, the number of all possible topological relations between each pair of objects in each space is equal or possibly less than the number in Table 2.

Table 2. Numbers of the derived patterns of the $9^{+}$-intersection matrix for every possible pair of basic objects embedded in $\mathbf{R}^{1}, \mathbf{R}^{2}, \mathbf{R}^{3}, \mathbf{S}^{1}$, and $\mathbf{S}^{2}$.

\begin{tabular}{cccccc}
\hline & $\mathbf{R}^{1}$ & $\mathbf{R}^{2}$ & $\mathbf{R}^{3}$ & $\mathbf{S}^{1}$ & $\mathbf{S}^{2}$ \\
\hline Point-Point & 6 & 2 & 2 & 2 & 2 \\
Point-DLine & 10 & 4 & 4 & 4 & 4 \\
Point-Region & - & 3 & 3 & - & 3 \\
Point-Body & - & - & 3 & - & - \\
DLine-DLine & 26 & 80 & 80 & 28 & 80 \\
DLine-Region & - & 26 & 45 & - & 26 \\
DLine-Body & - & - & 26 & - & - \\
Region-Region & - & 8 & 43 & - & 11 \\
Region-Body & - & - & 19 & - & - \\
Body-Body & - & - & 8 & - & - \\
\hline
\end{tabular}

For point-point relations in $\mathbf{R}^{2}, \mathbf{R}^{3}, \mathbf{S}^{1}$, and $\mathbf{S}^{2}$, the same two patterns of the $9^{+}$intersection matrix are derived. These two matrix patterns correspond to the two scenarios - two points coincide or not. Meanwhile, point-point relations in $\mathbf{R}^{1}$ yielded a larger number of matrix patterns, because in $\mathbf{R}^{1}$ the exterior of each point is subdivided into two primitives (i.e., front and back exterior subparts) and their order influences the distinction of topological relations. Similarly, the same four matrix patterns are derived for point-DLine relations in $\mathbf{R}^{2}, \mathbf{R}^{3}, \mathbf{S}^{1}$, and $\mathbf{S}^{2}$, but not for those in $\mathbf{R}^{1}$. These four matrix patterns correspond to the four scenarios where the point is located at the DLine's interior, exterior, start-point, or end-point. For point-region relations in $\mathbf{R}^{2}, \mathbf{R}^{3}$, and $\mathbf{S}^{2}$ and point-body relations in $\mathbf{R}^{3}$, we derived the same three matrix patterns, which correspond to the three scenarios where the point is located at the interior, exterior, or boundary of the region/body.

For DLine-DLine relations in $\mathbf{R}^{1}$, we derived 26 patterns of the $9^{+}$-intersection matrix. These 26 patterns correspond to the 26 relations between two directed intervals in $\mathbf{R}^{1}$ [20], because directed intervals are essentially DLines.

For DLine-DLine relations in $\mathbf{R}^{2}, 80$ patterns of the $9^{+}$-intersection matrix are derived. Kurata and Egenhofer [5] identified only 68 DLine-DLine relations in $\mathbf{R}^{2}$, as they used an extension of the 4-intersection. They also speculated that additional 12 relations appear if they distinguish the collapsed configurations (i.e., the configurations where one DLine contains another DLine). Our 80 patterns of the $9^{+}$- 
intersection matrix successfully distinguish these $68+12=80$ DLine-DLine relations.

For DLine-region relations in $\mathbf{R}^{2}$, we derived 26 patterns of the $9^{+}$-intersection matrix. This successfully corresponds to the result in [6], which identified 26 DLineregion relations based on the $9^{+}$-intersection.

DLine-DLine relations in $\mathbf{R}^{2}, \mathbf{R}^{3}$, and $\mathbf{S}^{2}$ yielded the same 80 matrix patterns. This result indicates that the set of 80 DLine-DLine relations in $\mathbf{R}^{2}$ are also seen in $\mathbf{R}^{3}$ and $\mathbf{S}^{2}$. Similarly, from the result that DLine-region relations in $\mathbf{R}^{2}$, DLine-region relations in $\mathbf{S}^{2}$, and DLine-body relations in $\mathbf{R}^{3}$ has yielded the same 26 matrix patterns, it is concluded that the set of 26 DLine-region relations in $\mathbf{R}^{2}$ are also seen in $\mathbf{S}^{2}$, and have a one-to-one correspondence with DLine-body relations in $\mathbf{R}^{3}$. This correspondence stems from the structural similarity between a region in $\mathbf{R}^{2}$ and a body in $\mathbf{R}^{3}$ (Fig. 2).

For DLine-Region relations in $\mathbf{R}^{3}$, we derived 45 patterns of the $9^{+}$-intersection matrix, among which 26 patterns are identical to the 26 matrix patterns derived for DLine-Region relations in $\mathbf{R}^{2}$. We confirmed that each of the remaining 19 patterns has at least one geometrical realization in $\mathbf{R}^{3}$ (Fig. 4). Consequently, we identified that topological DLine-Region relations in $\mathbf{R}^{3}$ consist of 19 relations peculiar to $\mathbf{R}^{3}$ (Fig. 4) and 26 relations common to $\mathbf{R}^{2}, \mathbf{R}^{3}$, and $\mathbf{S}^{2}$ (see [6] for the list). Such DLineRegion relations in $\mathbf{R}^{3}$ are useful for categorizing the movement patterns in association with region-like landmarks - for instance, how a bird moves around a pond. The newly identified 19 relations capture the bird's movement patterns that realize only in a three-dimensional space.
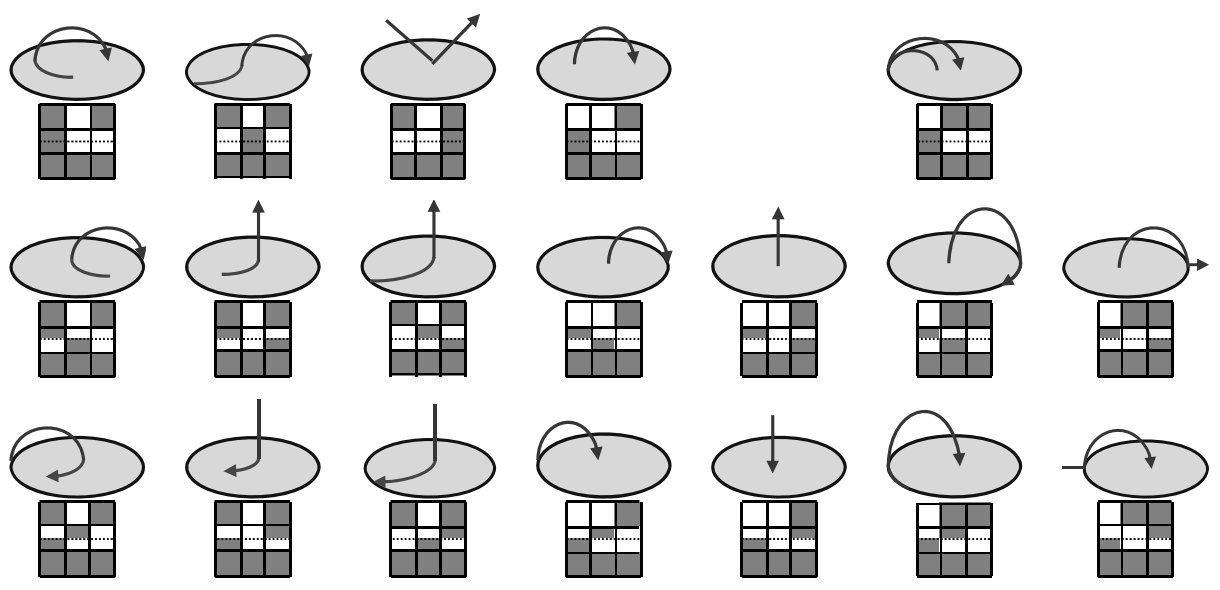

Fig. 4. 19 topological DLine-region relations, which are peculiar to $\mathbf{R}^{3}$.

For region-region relations in $\mathbf{R}^{2}$, eight patterns of the $9^{+}$-intersection matrix are derived. In this case, the matrix is essentially the 9-intersection matrix, because no topological part of a region is subdivided into multiple primitives. These eight matrix patterns are exactly the same as those derived in [2]. Similarly, the following correspondences to the findings of the previous studies are observed: 
- the eleven matrix patterns derived for region-region relations in $\mathbf{S}^{2}$ are the same as those derived in [11];

- the nineteen matrix patterns derived for region-body in $\mathbf{R}^{3}$ are the same as those derived in [9]; and

- the eight matrix patterns derived for body-body relations in $\mathbf{R}^{3}$ are the same as those derived in [9].

Meanwhile, for region-region relations in $\mathbf{R}^{3}$, we derived 43 patterns of the 9intersection matrix, while only 38 patterns are reported in [9]. Through the comparison the matrix patterns, we identified five missing relations (Fig. 5). In [21], 43 region-region relations in $\mathbf{R}^{3}$ are derived based on another spatial model, called Dimensional Model [18]. The 43 region-region relations in the Dimensional Model are equivalent to the region-region relations characterized by our 43 patterns of the 9intersection matrix.
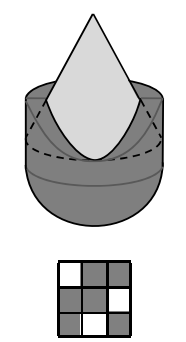
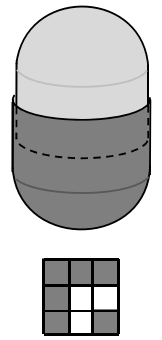
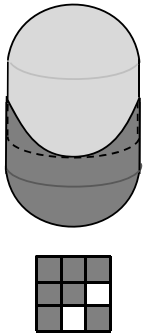

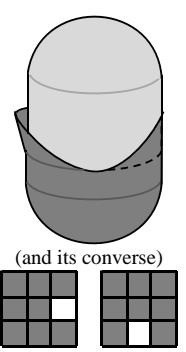

Fig. 5. Five topological region-region relations in $\mathbf{R}^{3}$ that should be added to the list in [9].

\section{Topological Relations Derived by Matrix Conversion}

A unique feature of the $9^{+}$-intersection is that the $9^{+}$-intersection matrix can be converted to the 9-intersection matrix, simply by integrating the elements of its nine element sets by union operation (Fig. 6). If the original $9^{+}$-intersection matrix captures the topological relation between a DLine and another object $X$, the 9-intersection matrix derived by the conversion captures the topological line- $X$ relations, as the distinction of the DLine's start-point and end-point is lost. Similarly, for the relations in $\mathbf{R}^{1}$, the distinction of objects' front and back exterior subparts is lost by the matrix conversion (Fig. 6b). Making use of this matrix conversion, the sets of candidates for topological line- $X$ relations and the modified candidate sets for topological relations in $\mathbf{R}^{1}$ are derived from the matrix patterns derived in Section 4 . Table 3 shows the number of the derived patterns of the 9-intersection matrix. 


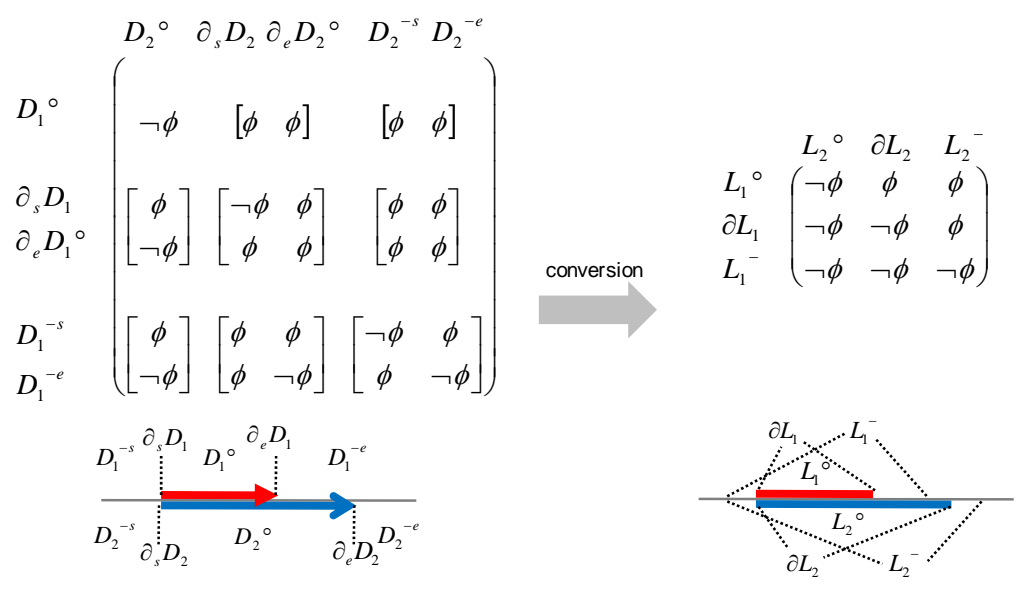

Fig. 6. Conversion from the $9^{+}$-intersection matrix to the 9-intersection matrix. In the left figure, DLines $D_{1}$ and $D_{2}$ are represented by two arrows.

Table 3. Numbers of the patterns of the 9-intersection matrix derived by matrix conversion.

\begin{tabular}{cccccc}
\hline & $\mathbf{R}^{1}$ & $\mathbf{R}^{2}$ & $\mathbf{R}^{3}$ & $\mathbf{S}^{1}$ & $\mathbf{S}^{2}$ \\
\hline Point-Point & 2 & - & - & - & - \\
Point-Line & 3 & - & - & - & - \\
Line-Line & 8 & 33 & 33 & 11 & 33 \\
Line-Region & - & 19 & 31 & - & 19 \\
Line-Body & - & - & 19 & - & - \\
\hline
\end{tabular}

For line-line relations in $\mathbf{R}^{1}$, eight patterns of the 9-intersection matrix are derived. These eight patterns correspond to the eight line-line relations in $\mathbf{R}^{1}$, identified in [22]. In addition, these eight matrix patterns are the same as the matrix patterns for region-region relations in $\mathbf{R}^{2}$. This indicates a one-to-one correspondence between the eight line-line relations in $\mathbf{R}^{1}$ and the eight region-region relations in $\mathbf{R}^{2}$. Similarly, we found a one-to-one correspondence between the eleven line-line relations in $\mathbf{S}^{1}$ and the eleven region-region relations in $\mathbf{S}^{2}$.

For line-line and line-region relations in $\mathbf{R}^{2}$, we derived 33 and 19 patterns of the 9-intersection matrix. These two sets of matrix patterns are the same as those in [2]. Similarly, for line-line, line-region, and line-body relations in $\mathbf{R}^{3}$, we derived 33,31 , and 19 patterns of the 9-intersection matrix, which are the same as those in [9].

The $9^{+}$-intersection matrix allows another type of matrix conversion when both objects have topological parts that can be subdivided into multiple primitives. This conversion removes the subdivision of topological parts with respect to only one of the two objects (Fig. 7). Accordingly, the matrix derived by the conversion is still the $9^{+}$-intersection matrix. Making use of this matrix conversion, we derived additional sets of patterns of the $9^{+}$-intersection matrix from those of the $9^{+}$-intersection matrix in Section 4 . Table 4 shows the numbers of the derived matrix patterns. 

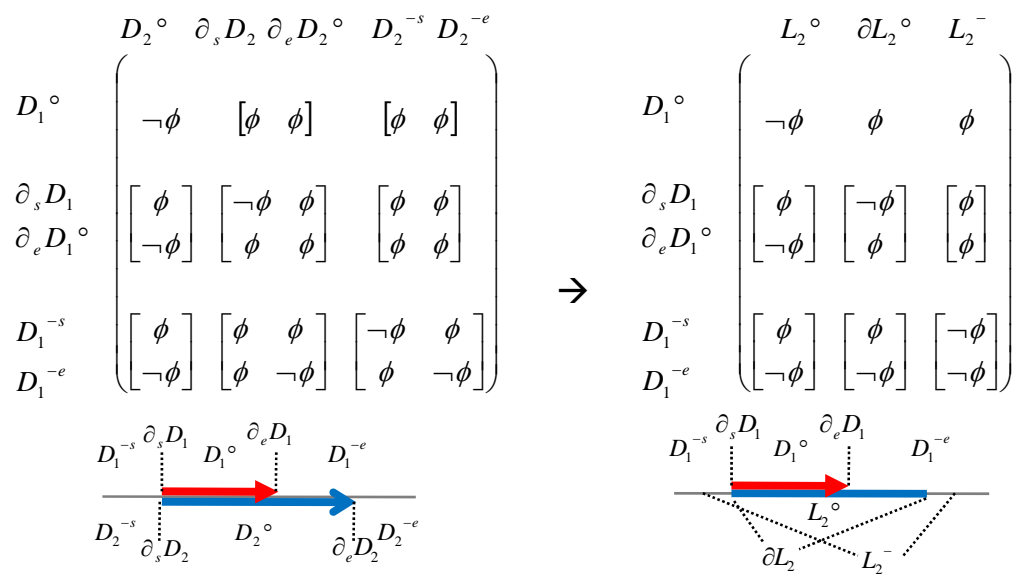

Fig. 7. Conversion of the $9^{+}$-intersection matrix that removes the subdivision of the second object's topological parts.

Table 4. Numbers of the patterns of the $9^{+}$-intersection matrix that are newly derived by the matrix conversion in Fig. 7.

\begin{tabular}{cccccc}
\hline & $\mathbf{R}^{1}$ & $\mathbf{R}^{2}$ & $\mathbf{R}^{3}$ & $\mathbf{S}^{1}$ & $\mathbf{S}^{2}$ \\
\hline Point-Point & 3 & - & - & - & - \\
Point-Line & 5 & - & - & - & - \\
DLine-Line & 13 & 49 & 49 & 16 & 49 \\
\hline
\end{tabular}

For point-point relations in $\mathbf{R}^{1}$, three patterns of the $9^{+}$-intersection matrix are derived. These three matrix patterns correspond to the three scenarios where one point precedes, coincides, or succeeds another point on the same axis. In the previous step, we derived only two patterns for the same relations, because the 9-intersection matrix does not distinguish whether one point precedes or succeeds another point. For the same reason, the number of the patterns of the $9^{+}$-intersection matrix derived for point-line relation in $\mathbf{R}^{1}$ is larger than that of the 9-intersection matrix.

For DLine-line relations in $\mathbf{R}^{1}, 13$ patterns of the $9^{+}$-intersection matrix are derived. Interestingly, these 13 matrix patterns correspond to the 13 interval relations in Allen's interval algebra [8], because DLine-Line relations in $\mathbf{R}^{1}$ essentially illustrate how one line (interval) extends with respect to another line (interval) on the same axis with the distinction of front and back. Similarly, for DLine-Line relations in $\mathbf{S}^{1}$, we derived 16 matrix patterns, which correspond to the 16 interval relations in a cyclic temporal frame identified in [10].

For DLine-line relations in $\mathbf{R}^{2}, \mathbf{R}^{3}$, and $\mathbf{S}^{2}$, the same 49 patterns of the $9^{+}$intersection matrix are derived. We confirmed that each of these 49 matrix patterns has at least one geometric realization (Fig. 8). Thus, it is concluded that these 49 matrix patterns represent the set of all possible DLine-line relations in $\mathbf{R}^{2}, \mathbf{R}^{3}$, and $\mathbf{S}^{2}$ under the $9^{+}$-intersection. Such DLine-line relations are useful for categorizing the movement patterns associated with a linear landmark, such as a wall or a trench. 


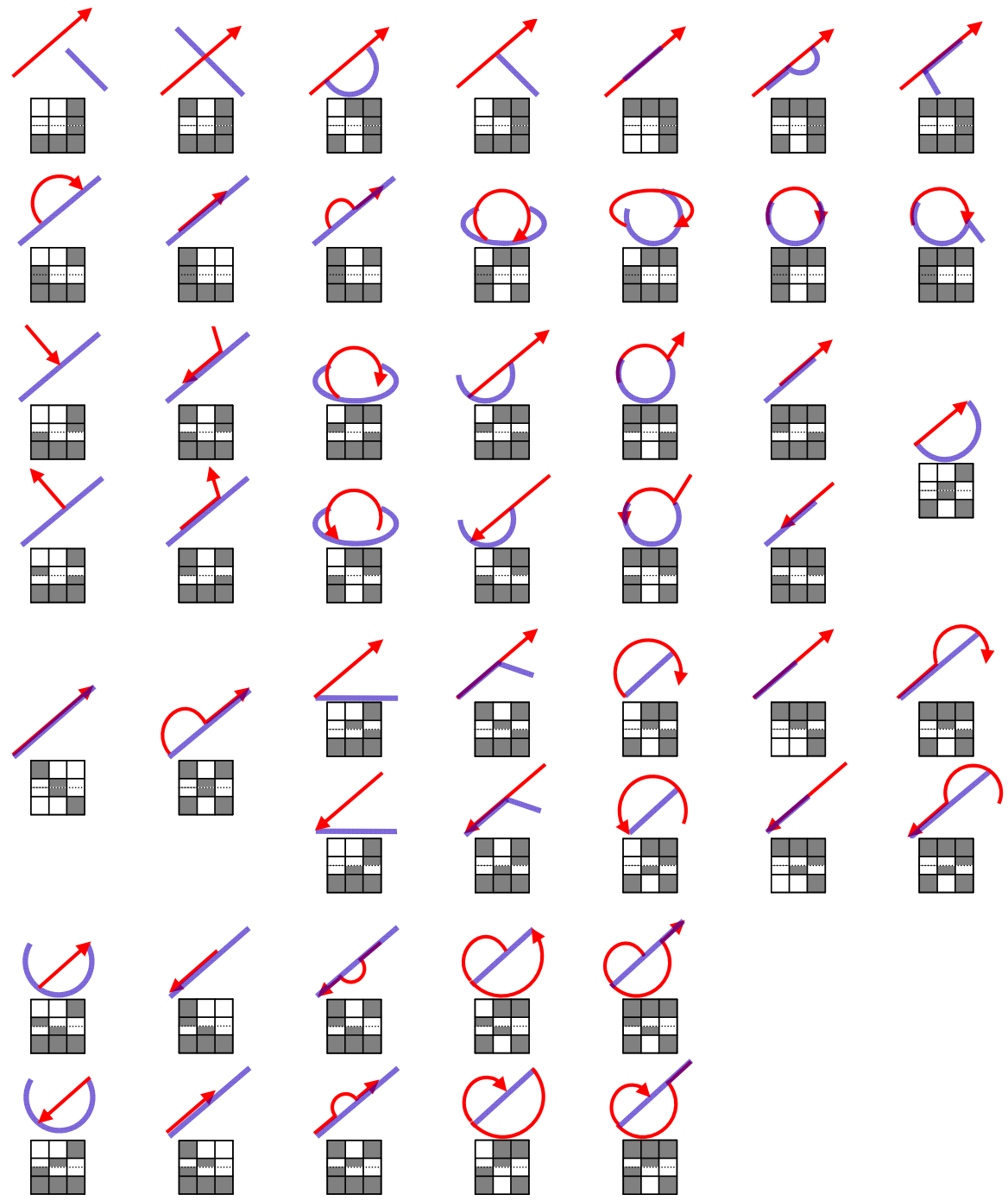

Fig. 8. 49 topological DLine-line relations, common to $\mathbf{R}^{2}, \mathbf{R}^{3}$, and $\mathbf{S}^{2}$, each with an example of geometric realizations.

Finally, through the comparison of Tables 2-4, we found the following features:

- The number of matrix patterns for line-line/DLine-line/DLine-DLine relations in $\mathbf{S}^{1}$ are larger than that for the counterparts in $\mathbf{R}^{1}$, because $\mathbf{S}^{1}$ allows the relation where one line/DLine includes the entire exterior of another line/DLine, but $\mathbf{R}^{1}$ does not.

- The number of topological relations between two spatial objects is invariant to the embedding space, as long as these two objects are lower dimensional than the 
space. One exception is point-point relations in $\mathbf{R}^{1}$ distinguished by the $9^{+}$intersection (Table 2), because the distinction of the points' front and back exteriors increases the number of point-point relations.

\section{Deriving Topological Relations related to Complicated Objects}

The combination of the $9^{+}$-intersection and the universal constraints are effective also for deriving the possible topological relations between a basic object and a more complicated object, or even the relations between two complicated objects (here, complicated objects mean the objects that are derived by a set operation on multiple basic objects). As a demonstration, this section derives all possible topological relations between a simple region (so far we have called this a region) and a simple region with a hole. For simplification, these relations are called topological regionregion $^{+1 \mathrm{H}}$ relations. In [7], 23 topological region-region ${ }^{+1 \mathrm{H}}$ relations in $\mathbf{R}^{2}$ are already identified, but those in $\mathbf{S}^{2}$ are not yet.

A simple region with a hole $X$ is defined as the difference between two simple regions $X^{*}$ and $X_{H}$, where $X^{*}$ contains $X_{H}$ entirely. $X^{*}$ is called $X^{\prime}$ 's generalized region, while $X_{H}$ is called $X$ 's hole [23]. A simple region with a hole in $\mathbf{S}^{2}$ may represent a ring-like object on a sphere (Fig. 9a) or a belt-like object that surrounds the sphere (Fig. 9b) Accordingly, region-region ${ }^{+1 \mathrm{H}}$ relations in $\mathbf{S}^{2}$ can be used for modeling, for instance, the spatial arrangement of an island and a typhoon, that of the iris and the covered surface of a human eye, or that of the Earth's surface receiving sunlight and a certain latitude zone.

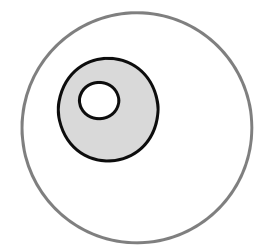

(a)

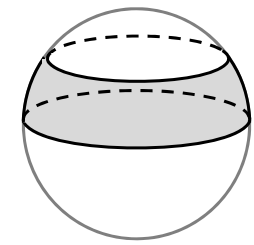

(b)

Fig. 9. Examples of simple regions with a hole embedded in $\mathbf{S}^{2}$.

The topological relation between a simple region $A$ and a simple region with a hole $B$ are characterized by the $9^{+}$-intersection matrix in Eqn. 4, where $\partial_{1} B, \partial_{2} B, B^{-1}$, and $B^{-2}$ are $B^{\prime}$ 's outer boundary, hole-side boundary, outer exterior, and hole-side exterior, respectively. In $\mathbf{S}^{2}$, the outer exterior and the hole-side exterior have no geometric difference (Fig. 9b) and, accordingly, their distinction depends on the observer's view.

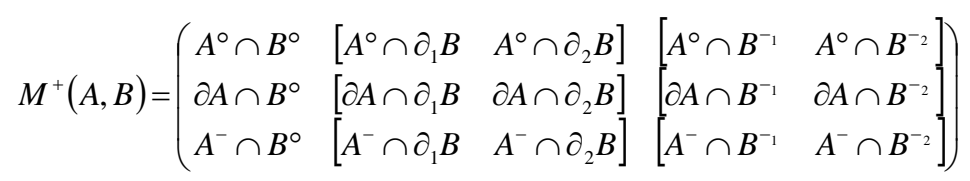


Instead of the 9+-intersection matrix in Eqn. 4, the same topological regionregion $^{+1 \mathrm{H}}$ relation is characterized by a pair of the 9-intersection matrices, which represent the topological region-region relation between $A$ and $B$ 's generalized region $B^{*}$ and that between $A$ and $B$ 's hole $B_{H}$, respectively $[7,23]$. The patterns of these two 9 -intersection matrices are determined uniquely from the pattern of the corresponding $9^{+}$-intersection matrix (Fig. 10).

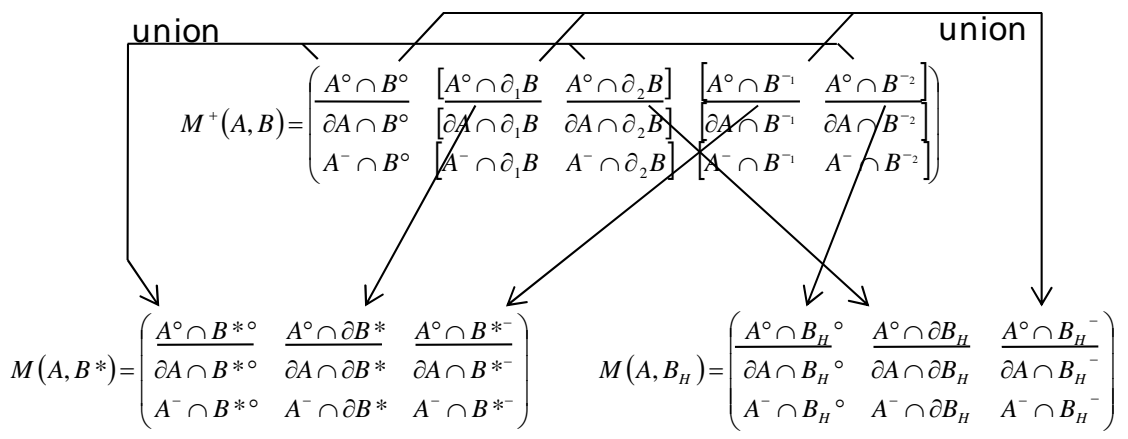

Fig. 10. Conversion from the $9^{+}$-intersection matrix for a topological region-region ${ }^{+1 \mathrm{H}}$ relation to a pair of the 9-intersection matrices. This figure shows the conversion of the elements in the matrix's first row. The elements in the second and third rows are converted in the same way.

Fig. 11 shows the topological structures of simple regions and simple regions with a hole embedded in $\mathbf{R}^{2}$ and $\mathbf{S}^{2}$. Based on this structural information and Conditions 19 (i.e., universal constraints), we computationally derived 23 and 37 matrix patterns for topological region-region ${ }^{+1 H}$ relations in $\mathbf{R}^{2}$ and $\mathbf{S}^{2}$, respectively. It is confirmed by the matrix conversion in Fig. 10 that the former 23 matrix patterns perfectly correspond to the 23 region-region ${ }^{+1 \mathrm{H}}$ relations in $\mathbf{R}^{2}$ identified in [7]. Among the latter 37 matrix patterns, 23 patterns are the same as those for region-region ${ }^{+1 \mathrm{H}}$ relations in $\mathbf{R}^{2}$. Meanwhile, we confirmed that each of the remaining 14 matrix patterns has at least one geometric realization in $\mathbf{S}^{2}$ (Fig. 12). Thus, it is confirmed that topological region-region ${ }^{+1 \mathrm{H}}$ relations in $\mathbf{S}^{2}$ consist of 14 relations peculiar to $\mathbf{S}^{2}$ (Fig. 12) and 23 relations that are also seen in $\mathbf{R}^{2}$ (see [7] for the list).

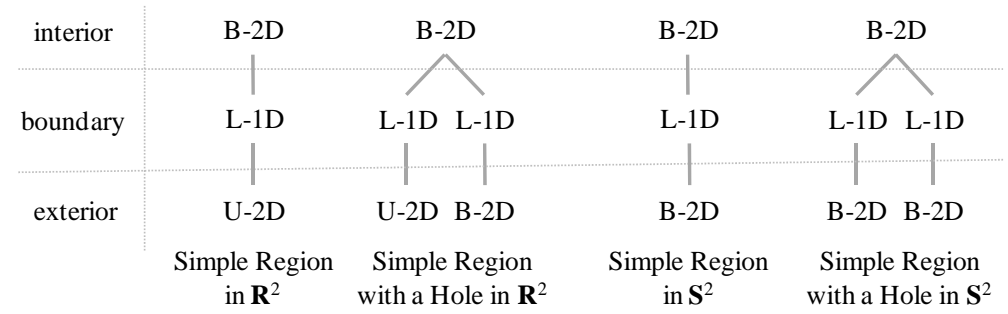

Fig. 11. Topological structures of simple regions and simple regions with a hole, embedded in $\mathbf{R}^{2}$ and $\mathbf{S}^{2}$. 


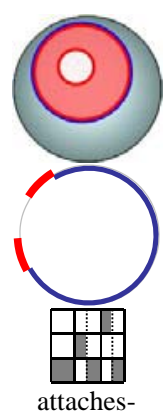

disjoint

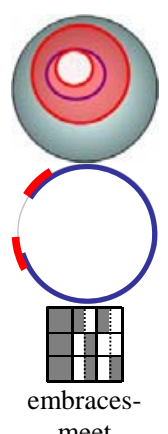

meet

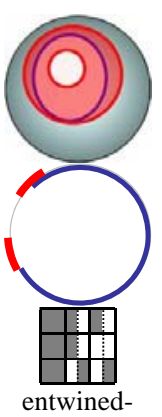

disjoint
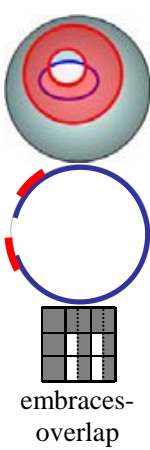

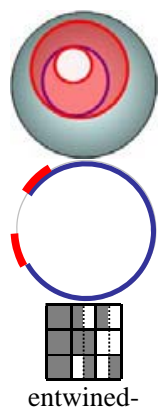

meet
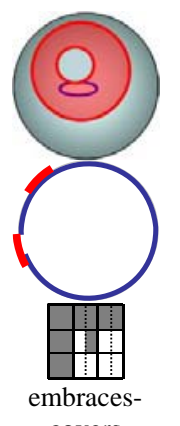
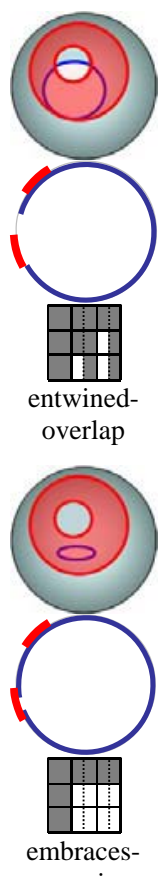

contains

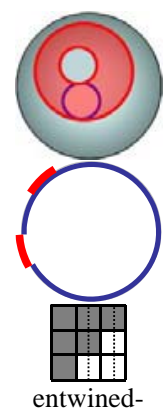

covers
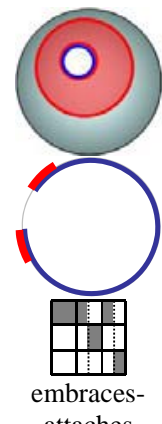

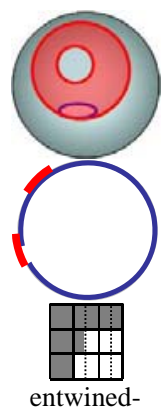

contains
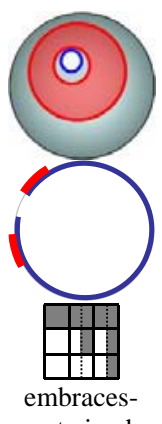

entwined

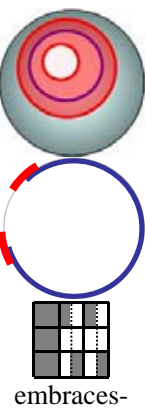

disjoint

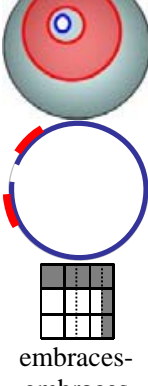

Fig. 12. 14 topological relations between a region $A$ and a region with a hole $B$, which are peculiar to $S^{2}$, together with an example configuration and its cross section. The name given to each relation combines the name of the topological relation between $A$ and $B$ 's generalized region and that between $A$ and $B$ 's hole, following the notation of [7].

In the same way, it is possible to derive the candidates for topological relations between a basic object and a complicated object (e.g., DLine-region ${ }^{+1 \mathrm{H}}$ relations), as well as the relations between two complicated objects (e.g., region ${ }^{+1 \mathrm{H}}$-region $^{+1 \mathrm{H}}$ relations).

\section{Conclusions}

Topological relations, which concern how two objects intersect with each other, have been studied for decades in pursuit of cognitively adequate models of the objects' spatial arrangement. The $9^{+}$-intersection captures such topological relations in a systematic way using a nested matrix, called the $9^{+}$-intersection matrix. This paper developed a set of universal constraints on the patterns of the $9^{+}$-intersection matrix. These constraints allow us to derive the candidates for the topological relations between a given pair of objects, regardless of their geometric types. The combination of the $9^{+}$-intersection and the universal constraints is, therefore, highly useful when we study new sets of topological relations. Indeed, we newly identified DLine-Region relations in $\mathbf{R}^{3}$, DLine-Line relations in $\mathbf{R}^{2}, \mathbf{R}^{3}$, and $\mathbf{S}^{2}$, and region-region ${ }^{+1 \mathrm{H}}$ relations 
in $\mathbf{S}^{2}$, as well as found that many sets of topological relations in $\mathbf{S}^{1}$ and $\mathbf{S}^{2}$ are equivalent to those in $\mathbf{R}^{1}$ and $\mathbf{R}^{2}$.

A remaining issue is to answer whether our universal constraints are always sufficient, in the sense that every pattern of the $9^{+}$-intersection matrix, determined by the universal constraints, always has at least one geometric realization. The constraints' sufficiency is empirically confirmed with respect to the relation between every possible pair of basic objects embedded in $\mathbf{R}^{1}, \mathbf{R}^{2}, \mathbf{R}^{3}, \mathbf{S}^{1}$, and $\mathbf{S}^{2}$. For the relations between complicated objects, however, it becomes hard to check the validity of all derived matrix patterns due to its large number. Thus, a mathematical examination of the constraints' sufficiency is left for future work.

\section{Acknowledgements}

Yohei Kurata's work is supported by DFG (Deutsche Forschungsgemeinschaft) through the Collaborative Research Center SFB/TR 8 Spatial Cognition. The author appreciates Dr. Max J. Egenhofer, Dr. Roland Billen, and Dr. Tetsushi Ito for their helpful advice and information.

\section{References}

1. Egenhofer, M., Franzosa, R.: Point-Set Topological Spatial Relations. International Journal of Geographical Information Systems 5, 161-174 (1991)

2. Egenhofer, M., Herring, J.: Categorizing Binary Topological Relationships between Regions, Lines and Points in Geographic Databases. In: Egenhofer, M., Herring, J., Smith, T., Park, K. (eds.): NCGIA Technical Reports 91-7. National Center for Geographic Information and Analysis, Santa Barbara, CA, USA (1991)

3. Randell, D., Cui, Z., Cohn, A.: A Spatial Logic Based on Regions and Connection. In: Nebel, B., Rich, C., Swarout, W. (eds.): 3rd International Conference on Knowledge Representation and Reasoning, pp. 165-176. Morgan Kaufmann (1992)

4. Schneider, M., Behr, T.: Topological Relationships between Complex Spatial Objects. ACM Transactions on Database Systems 31, 39-81 (2006)

5. Kurata, Y., Egenhofer, M.: The Head-Body-Tail Intersection for Spatial Relations between Directed Line Segments. In: Raubal, M., Miller, H., Frank, A., Goodchild, M. (eds.): GIScience'06, LNCS, vol. 4197, pp. 269-286. Springer (2006)

6. Kurata, Y., Egenhofer, M.: The $9^{+}$-Intersection for Topological Relations between a Directed Line Segment and a Region. In: Gottfried, B. (ed.): 1st International Symposium for Behavioral Monitoring and Interpretation, pp. 62-76 (2007)

7. Egenhofer, M., Vasardani, M.: Spatial Reasoning with a Hole. In: Winter, S., Duckham, M., Kulik, L., Kuijpers, B. (eds.): COSIT'07, LNCS, vol. 4736, pp. 303-320. Springer (2007)

8. Allen, J.: An Interval-Based Representation of Temporal Knowledge. In: Hayes, P. (ed.): 7th International Joint Conference on Artificial Intelligence, pp. 221-226 (1981)

9. Zlatanova, S.: On 3D Topological Relationships. In: 11th International Workshop on Database and Expert Systems Applications, pp. 913-924. IEEE Computer Society (2000)

10.Hornsby, K., Egenhofer, M., Hayes, P.: Modeling Cyclic Change. In: Chen, P., Embley, D., Kouloumdjian, J., Liddle, S., Roddick, J. (eds.): Advances in Conceptual Modeling, LNCS, vol. 1227, pp. 98-109. Springer (1999)

11.Egenhofer, M.: Spherical Topological Relations. Journal on Data Semantics III 25-49 (2005) 
12.Egenhofer, M., Sharma, J.: Topological Relations between Regions in $\mathbf{R}^{2}$ and $\mathbf{Z}^{2}$. In: Abel, D., Ooi, B.C. (eds.): Advances in Spatial Databases: 3rd International Symposium on Large Spatial Databases, LNCS, vol. 692, pp. 316-336. Springer (1993)

13.Mark, D.: Calibrating the Meanings of Spatial Predicates from Natural Language: LineRegion Relations. In: Waugh, T., Healey, R. (eds.): 6th International Symposium on Spatial Data Handling, pp. 538-553. Taylor Francis (1994)

14.Nedas, K., Egenhofer, M., Wilmsen, D.: Metric Details of Topological Line-Line Relations. International Journal of Geographical Information Science 21, 21-48 (2007)

15.Clementini, E., Di Felice, P.: A Model for Representing Topological Relationships between Complex Geometric Features in Spatial Databases. Information Science 90, 121-136 (1996)

16.Alexandroff, P.: Elementary Concepts of Topology. Dover Publications, Mineola, NY (1961)

17.Egenhofer, M., Franzosa, R.: On the Equivalence of Topological Relations International Journal of Geographical Information Systems 9, 133-152 (1995)

18.Billen, R., Zlatanova, S., Mathonet, P., Boniver, F.: The Dimensional Model: A Framework to Distinguish Spatial Relationships. In: Richardson, D., van Oosterom, P. (eds.): 10th International Symposium on Spatial Data Handling, pp. 285-298. Springer (2002)

19.Mark, D., Egenhofer, M.: Modeling Spatial Relations between Lines and Regions: Combining Formal Mathematical Models and Human Subjects Testing. Cartography and Geographical Information Systems 21, 195-212 (1994)

20.Renz, J.: A Spatial Odyssey of the Interval Algebra: 1. Directed Intervals. In: Nebel, B. (ed.): 7th International Joint Conference on Artificial Intelligence, pp. 51-56. Morgan Kaufmann (2001)

21.Billen, R.: Nouvelle Perception De La Spatialité Des Objets Et De Leurs Relations. Développment D'une Modélisation Tridimensionnelle De L'information Spatiale. Department of Geography, Ph.D. Thesis. University of Liège, Liège, Belgium (2002)

22.Pullar, D., Egenhofer, M.: Towards Formal Definitions of Topological Relations among Spatial Objects. In: Marble, D. (ed.): 3rd International Symposium on Spatial Data Handling, pp. 225-241 (1988)

23.Egenhofer, M., Clementini, E., Di Felice, P.: Topological Relations between Regions with Holes. International Journal of Geographical Information Science 8, 129-142 (1994)

\section{Appendix: Proofs of Conditions 1-9 in Section 3}

\section{Conditions 1-3:}

These three conditions are satisfied because the primitives of a spatial object are jointly exhaustive, mutually exclusive, and self-connected, respectively.

\section{Conditions 4-5:}

These two conditions are basic properties of topology.

\section{Condition 6:}

Let $P_{k}$ be a $P_{i}$ 's adjacent higher-dimensional primitive. $P_{k}$ is adjacent to $P_{i} \cap Q_{j}$ as well. On the other hand, $P_{i} \cap Q_{j}$ is surrounded entirely by the set of all $Q_{j}$ 's adjacent higher-dimensional primitives and $Q_{j} \backslash P_{i}$. Accordingly, $P_{k}$ intersects with at least one of $Q_{j}$ 's adjacent higher-dimensional primitives or $Q_{j}$. 


\section{Condition 7:}

Let us consider a subspace of $S$, called $S^{\prime}$, which embeds $Q_{j}$ and whose dimension is the same with $Q_{j}$. Since $Q_{j}$ contains $P_{i}, S^{\prime}$ also embeds $P_{i}$. $P_{i}$ 's adjacent primitives in $S^{\prime}$, which are always lower-dimensional than $P_{i}$, is identical with the set of $P_{i}$ 's adjacent lower-dimensional primitives in $S$. Since $Q_{j}$ contains $P_{i}$ in $S^{\prime}, Q_{j}$ intersect with at least one of $P_{i}$ 's adjacent primitives in $S^{\prime}$. Accordingly, $Q_{j}$ intersect with at least one of $P_{i}$ 's adjacent lower-dimensional primitives in $S$.

\section{Condition 8:}

Let $\boldsymbol{Q}$ be the set of all of $Q_{j}$ 's adjacent higher-dimensional primitives. $P_{i} \cap Q_{j}$ is surrounded entirely by $\boldsymbol{Q}$ and $Q_{j} \backslash P_{i}$. Accordingly, $P_{i}$, which contains $P_{i} \cap Q_{j}$ and higher dimensional than $Q_{j} \backslash P_{i}$, intersects with at least one of primitives in $\boldsymbol{Q}$. Let us assume that $Q_{j}$ has an adjacent primitive that is bounded, non-looped, and onedimensionally higher than $Q_{j}$, called $Q_{k}$. $Q_{k}$ is an element of $\boldsymbol{Q}$. Let $S^{\prime}$ be a sub-space of $S$, which embeds $P_{i}$ and whose dimension is the same with $P_{i}$. Since $Q_{j}$ cannot split the $Q_{k}, Q_{j}$ must be located at the end of $Q_{k}$. Accordingly, $P_{i} \cap Q_{j}$ is not surrounded entirely by $Q_{k}$ in $S^{\prime}$, but by the combination of $Q_{k}$, some other elements in $Q$, and $Q_{j} \backslash P_{i}$ in $S^{\prime}$. On the other hand, $P_{i} \cap Q_{j}$ is surrounded entirely by $P_{i} \backslash Q_{j}$ and $Q_{j} \backslash P_{i}$ in $S^{\prime}$. Consequently, if $Q_{k}$ exists, $P_{i}$ intersects with not only $Q_{k}$, but also another element in $\boldsymbol{Q}$.

\section{Condition 9:}

Let $P_{i}$ be $A$ 's only one unbounded primitive whose dimension is the same with $S$. Since $P_{i}$ 's complement $\bar{P}_{i}$ is bounded, each of $B$ 's unbounded primitive is not entirely included in $\bar{P}_{i}$. Consequently, $P_{i}$ intersects with $B$ 's unbounded primitives. Note $P_{\mathrm{i}}$ refers to the exterior of a spatial object in $\mathbf{R}^{n}(n \geq 2)$. 